\title{
STUDY ON COMPOUND GENETIC AND BACK PROPAGATION ALGORITHM FOR PREDICTION OF COAL AND GAS OUTBURST RISK
}

\author{
Yaqin Wu', Kai Wang ${ }^{2}$, Maoguang Wang ${ }^{1,3}$ \\ 'School of Computer Science \& Technology, China University of Mining and Technology, \\ Xuzhou 221008, Jiangsu, China
}

${ }^{2}$ School of Mining \& Safety Engineering, China University of Mining and Technology, Xuthou 221008, Jiangsu, China

${ }^{3}$ Institute of Computing Technology, Chinese Academy of Sciences, Beijing 100080, China

Abstract: $\quad$ Coal and gas outburst is a very complex phenomenon of dynamic disaster in coal mine. There exists a complex non-linear mapping relationship which could not be described with functions between outburst risk and its influential factors. Due to the originality and superiority of artificial neural network (ANN) for modeling and imitating non-linear problems, an ANN model for prediction of outburst risk is set up. Then through practical application, the performance of commonly applied Back Propagation (BP) network for outburst risk prediction is analyzed. Aimed at the weakness of $B P$ algorithm and based on the overall searching characteristic of Genetic Algorithm (GA), an improved compound GA-BP algorithm is used to optimize the model, then both the performance of the network and the predicting reliability of the model are improved.

Key words: outburst risk prediction, artificial neural network, compound GA-BP algorithm

Please use the following format when citing this chapter:

Wu, Y., Wang, K., Wang, M., 2006, in IFIP International Federation for Information Processing. Volume 228, Intelligent Information Processing III, eds. Z. Shi, Shimohara K., Feng D., (Boston: Springer), pp. 233-241. 


\section{INTRODUCTION}

Coal and gas outburst (briefly named "outburst") is one of the biggest natural disasters which are seriously imperiling safe productivity of coal mine. Further studies of reliable outburst prediction methods are essential to effective prevention of outburst. It is known through numerous researches that the occurrence of outburst is a catastrophic behavior of a complex nonlinear dynamic system during its evolution in time and space, and there exists a complex non-linear mapping relationship which could not be described with functions between outburst risk and its influential factors ${ }^{[1]}$. Artificial neural network (ANN) possesses originality and superiority of modeling and imitating for dealing with such non-linear problems. Recently, the model of ANN has been tried to apply in outburst prediction and its effect has also been validated through applications. However ANN itself has some weakness. In this paper, through analyzing the performance of commonly applied Back Propagation (BP) neural network for outburst risk prediction, it is indicated that there exists some weakness of BP algorithm such as easily falling into local extremum, slow speed of convergence and vibrating effect, especially the result of BP algorithm is closely related to the initial status of the network. Consequently, in order to improve the perforemence of BP neural network, based on the overall searching characteristic of Genetic Algorithm (GA), an improved compound genetic and back propagation (GA-BP) algorithm is adopted to optimize the ANN model.

\section{DESCRIPTION OF THE PROBLEM}

Based on the synthetical hypothesis of outburst mechanism and statistical data from the spot, it can be drawn that the factors which control the occurrence and intensity of outburst mainly include: gas pressure (gas content), ground stress, coal mass strength and the thickness of soft coal seam. Moreover, the factors such as geological structure and pitch angle of coal seam, passage tunnel for outburst and the action of mining and drivage also influence the occurrence and intensity of outburst. Through simulating tests in laboratory of CUMT, the above first four factors are analyzed and their influence upon the possibility of outburst occurrence is investigated systematically $[1,2]$.

During the simulating tests, there occurred two typical dynamic phenomena which can be classified as: (I) non outburst risk, (II) outburst. Respectively using $\mathrm{P} 0, \mathrm{Pg}, \mathrm{Hm}, \mathrm{Sc}$ to represent ground stress, gas pressure, thickness of soft coal body and sieving modulus of coal mass, the concrete 
experimental conditions and results of the tests are listed in Table 1, where the strength of coal mass is expressed as sieving modulus. The larger sieving modulus is, the smaller coal mass strength is.

In this paper, the method of ANN will be adopted to set up the mapping relationship between outburst risk and $P_{0}, P_{g}, H_{\mathrm{m}}, S_{\mathrm{c}}$, so as to realize precise prediction of outburst risk.

Table l. Experiment data and results of outburst simulating tests

\begin{tabular}{|c|c|c|c|c|c|c|}
\hline No. & Site for Sampling & $P_{0} / \mathrm{MPa}$ & $\mathrm{Pg}_{\mathrm{g}} \mathrm{MPa}$ & $H_{m} / \mathrm{mm}$ & $S_{c}$ & Outburst Risk \\
\hline 1 & Luling 82 & 14.37 & 0.18 & 109.0 & 8.1 & 1 \\
\hline 2 & Luling 83 & 14.37 & 0.21 & 38.0 & 6.2 & I \\
\hline 3 & Kongzhuang $8 \#$ & 16.61 & 0.22 & 37.0 & 11.1 & I \\
\hline 4 & Jiahe $2 \#$ & 13.09 & 0.13 & 163.5 & 7.8 & I \\
\hline 5 & Jiahe 9\# & 17.24 & 0.21 & 85.5 & 14.7 & 1 \\
\hline 6 & Majiagou 9\# & 14.69 & 0.23 & 30.5 & 15.1 & I \\
\hline 7 & Jiulishan Y & 13.73 & 0.14 & 76.0 & 7.7 & 1 \\
\hline 8 & Jinggezhuang & 18.52 & 0.27 & 33.0 & 13.2 & $\mathrm{I}$ \\
\hline 9 & Panji No.1 Mine & 11.50 & 0.20 & 96.5 & 7.6 & 1 \\
\hline 10 & Panji No.3 Mine & 16.61 & 0.10 & 142.5 & 7.1 & 1 \\
\hline 11 & Jiaoxi Hard Coal & 14.05 & 0.32 & 105.0 & 4.9 & II \\
\hline 12 & Luling 83 & 14.37 & 0.72 & 85.5 & 6.2 & II \\
\hline 13 & Luling $9 \#$ & 15.01 & 0.44 & 79.0 & 11.2 & II \\
\hline 14 & Luling 9\# & 15.01 & 0.36 & 62.0 & 11.2 & II \\
\hline 15 & Luling 10\# & 13.09 & 0.35 & 60.0 & 7.3 & II \\
\hline 16 & Kongzhuang 8\# & 16.61 & 0.35 & 127.0 & 11.1 & II \\
\hline 17 & Jiahe $7 \#$ & 12.77 & 0.43 & 125.0 & 8.8 & II \\
\hline 18 & Majiagou 9\# & 14.69 & 0.48 & 68.0 & 15.1 & II \\
\hline 19 & Majiagou 12 & 11.50 & 0.52 & 33.5 & 12.1 & II \\
\hline 20 & Majiagou 12 & 11.50 & 0.55 & 67.0 & 12.1 & II \\
\hline 21 & Baishanping $6 \#$ & 13.73 & 0.22 & 34.5 & 6.8 & 11 \\
\hline 22 & Baishanping $6 \#$ & 13.73 & 0.41 & 34.5 & 6.8 & Il \\
\hline 23 & Jiulishan Y & 13.73 & 0.36 & 75.5 & 7.7 & II \\
\hline 24 & Xinzhuangzi & 14.37 & 0.48 & 75.5 & 8.7 & 11 \\
\hline 25 & Jinggezhuang & 18.52 & 0.25 & 118.5 & 13.2 & II \\
\hline 26 & Panji No. 1 Mine & 11.50 & 0.44 & 97.5 & 7.6 & 11 \\
\hline 27 & Panji No. 2 Mine & 13.41 & 0.49 & 73.0 & 12.5 & II \\
\hline 28 & Panji No. 2 Mine & 13.41 & 0.39 & 56.5 & 12.5 & 11 \\
\hline 29 & Panji No.3 Mine & 16.61 & 0.48 & 142.5 & 7.1 & II \\
\hline 30 & Jiaoxi Hard Coal & 14.05 & 0.74 & 105.0 & 4.9 & II \\
\hline 31 & Jiaoxi Soft Coal & 12.77 & 0.30 & 191.5 & 11.8 & II \\
\hline 32 & Jiaoxi Soft Coal & 12,77 & 0.75 & 74.5 & 11.8 & II \\
\hline 33 & Luling 82 & 14.37 & 0.65 & 110.0 & 8.1 & Il \\
\hline 34 & Luling 10\# & 13.10 & 0.85 & 172.0 & 7.3 & II \\
\hline 35 & Kongzhuang $7 \#$ & 17.24 & 0.82 & 118.0 & 5.9 & II \\
\hline 36 & Jiahe $2 \#$ & 13.36 & 0.82 & 162.5 & 7.8 & II \\
\hline 37 & Jiahe $7 \#$ & 12.77 & 1.0 & 37.0 & 8.8 & Il \\
\hline 38 & Majiagou 9\# & 14.69 & 1.16 & 30.5 & 15.1 & Il \\
\hline
\end{tabular}




\begin{tabular}{lllllll}
39 & Mixed Coal 1\# & 11.82 & 0.91 & 161.0 & 7.6 & II \\
40 & Mixed Coal 2\# & 19.48 & 0.61 & 107.5 & 6.6 & II \\
41 & Xinzhuangzi & 14.37 & 0.93 & 154.5 & 8.7 & II \\
\hline
\end{tabular}

\section{ANALYSIS ON THE ABILITY OF BP ALGORITHM FOR OUTBURST RISK PREDICTION}

\subsection{Back Propagation Neural Network}

In current applications of ANN, BP network model and its varied forms are usually mainly adopted because they represent the essence of ANN. BP network is a multi-layer forward network with unidirectional propagation, its construction is shown in Fig. ${ }^{[3,4]}$. BP network can be regarded as a highly non-linear mapping relationship between the input and output which can approximate complex function through compounding the simple non-linear functions time after time. It has been proved through theoretical research that $\mathrm{BP}$ network could realize any successive function at any due precision.

The learning process of BP network is a typically tutorial one realized by BP Algorithm which is the most popular training and learning method of ANN. The advantages of standard BP Algorithm based on gradient descending method and other improved forms lie in the accurate optimality searching and the ability of self-learning.
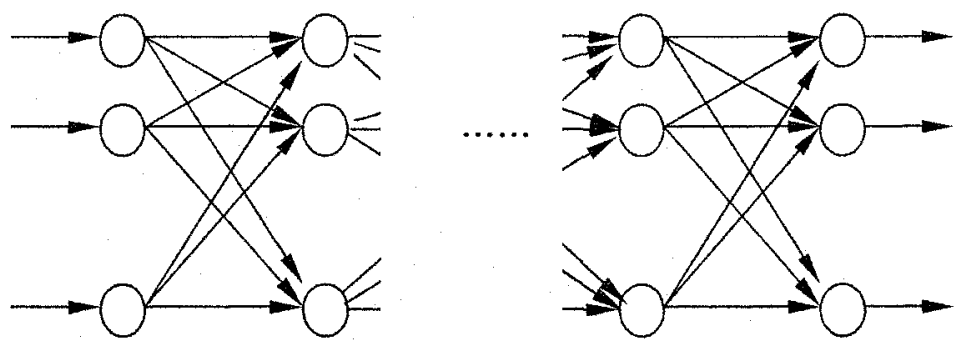

Figure I. BP network construction

\subsection{Establishment of the ANN Model}

In this paper, a three-layer BP neural network is set up. And there are four units in input layer which respectively represent the four influence 
factors of outburst. According to the test calculation results, it is determined that the network consists of two hidden layers of which the first one includes four units and the second one includes three units. The output layer includes one unit which represents outburst risk. The logarithmic tangent function is selected as the transferring function of the network between the input layer and the first hidden layer, the first hidden layer and the second hidden layer. The linear function is selected as the transferring function of the network between the second hidden layer and output layer. The calculating process of the network is programmed by MATLAB software. The function "trainlm" is selected as the training function and "learngdm" as the learning function. The sum-squared error function "sse" is selected as the training performance function of the network.

Firstly normalize the data of 41 samples in Table 1 through transforming all sample data into the number of which the average value is 0 and the standard error is 1 . Then analyze their main composition to get rid of the data whose variation is lower than 0.01 . The handled samples are divided into two groups among which $80 \%$ (33) samples are taken as the training samples and $20 \%$ (8) samples are taken as the predicting samples. The requirements for dividing groups are that both of the groups should include the two classifications of outburst risk, and the training samples should exclude predicting samples and contain the features of all samples. The training objective is $0.01^{2} \times 33=0.0033$.

\subsection{Analysis of the Training and Prediction Results}

According to the estabilished BP neural network model, the training and predicting process for outburst risk prediction has been conducted for many times. It is shown through analyzing every training and prediction results that there exist the following limitations of BP algorithm:

(1) The failure probability of network training is higher. The main reason is as follows. The goal of the network is trying to get the overall extremum of the complex non-linear function, however there usually exist several local extrema of such function, therefore as an optimizing method through local searching BP Algorithm is prone to fall into local extremum, converge slowly and even vibrate, so as to fail in training the network.

(2) The prediction result of the network is not so satisfied. There are two main reasons. Firstly, there exists contradictory between prediction ability and training ability of the network. Generally, the prediction ability of network will increase as its training ability improves, but there is an extreme limit of this trend beyond which the prediction ability of network will decrease as its training ability improves on the contrary. This is so-called 
"Over Fitness". Secondly, for all of the training processes, there is not only one final combination of weights and threshold values which satisfy the due training precision, so different combinations of weights (and threshold values) will lead to distinct prediction results.

\section{ANALYSIS OF COMPOUND GA-BP ALGORITHM AND ITS PREDICTION RESULT}

\subsection{Genetic Algorithm}

Genetic algorithm (GA) is basically based on natural biological evolution. A GA functions by generating an initial population from a random selection of possible solutions (which are analagous to chromosomes) to a given problem. It then evaluates each of those solutions, and a value for fitness is assigned to each solution (chromosome) depending on how close it actually is to solving the problem (thus arriving to the answer of the desired problem). Those chromosomes with a higher fitness value are more likely selected to reproduce new solutions (offspring, which can mutate after reproduction). The offspring is a product of the father and mother, whose composition consists of a combination of genes from them (this process is known as "crossing over"). If the new generation contains a solution that produces an output that is close enough or equal to the desired answer then the problem has been solved. If this is not the case, then the new generation will go through the same process as their parents did. This will continue until some condition (for example number of populations or improvement of the best solution) is satisfied ${ }^{[4,5]}$.

Compared with BP algorithm, Genetic algorithm (GA) possesses the following characteristics:

(1) The outstanding advantages of GA lie in that the gradient information of error function is unnecessary, and it is also unnecessarily considered whether the error function is differential or not. If it is easy to obtain the gradient information during the training process, perhaps BP algorithm will be superior to Genetic evolution algorithm in the speed of convergence.

(2) Both of the results of two algorithms are sensitive to their calculating parameters. Especially the result of BP algorithm is closely related to the initial status of the network. 
(3) As the optimizing process of objective function is concerned, GA is good at overall searching, on the contrary BP algorithm is more effective in local searching.

(4) Limited by encoding precision, sometimes GA is hard to reach higher training precision.

\subsection{Compound GA-BP Algorithm}

It can be known through the above analysis that both GA and BP algorithms have their own advantages and disadvantages. If combining their advantages, the better training results will be obtained. Because GA possesses very strong ability of overall searching for optimization of the objective function, and can get the overall optimal solution in higher probability, so that the weakness of BP algorithm can be overcome by using GA to search during the earlier stage. It is shown by some related researches that the training efficiency and effect of compound GA-BP algorithm are significantly improved compared with GA or BP algorithm alone.

In this paper, the compound GA-BP algorithm is used to realize the learning and predicting ability of the network, so that the training process of the network can be divided into two steps: firstly, optimizing the initial weights (and threshold values) of BP network by GA and then obtaining the overall optimal solution in a certain range; then using BP algorithm to correct the network weights (and threshold values) and acquire precise solution.

The concrete method for realizing compound GA-BP algorithm is as follows:

(1) Select the network construction and the learning rule, set up the network parameters.

(2) Estimate the solution space of the network weights (and threshold values) by the designed BP network, and preliminarily design the range of the solution space of the network weights (and threshold values).

(3) Compile the calculating program of fitness function. In this paper, the fitness function is determined as $e^{-F}$, among which $E$ is average square error.

(4) Select the parameters of GA. In this paper, the population size is 70, the gene encoding pattern is float code, the number of genetic generations is 500 , the crossover rate is 0.6 and the mutation rate is 0.05 .

(5) Use the operators of crossover, mutation and selection to complete the optimization of the network weights (and threshold values) until reaching the genetic objective. 
(6) Take the optimized weights (and threshold values) by GA as the initial weights (and threshold values) of the BP network, then use BP algorithm to conduct precise training.

(7) Training process will end if the due training precision can be satisfied after the sixth step, whereas go back to the fifth step until reaching the training objective.

\subsection{Analysis of the Training and Prediction Results}

The topologic network structure of the model is also 4-4-3-1. During the training process, the advantage of the compound GA-BP algorithm in the speed of convergence has been validated. In addition, it is shown from the training results of many times of calculations that the final combinations number and varying range of the network weights and threshold values trained by compound GA-BP algorithm are both less than those traned by $\mathrm{BP}$ algorithm, consequently the reliability of prediction result can be improved.

After the training process finished while the due training precision is satisfied, the weights between the input layer and the hidden layer, the hidden layer and the output layer, and the threshold values of every units are listed in Table 2 . The prediction results are shown in Table 3.

Table 2. The final network weights and threshold values trained by compound GA-BP algorithm

\begin{tabular}{llllll}
\hline & Weights & & & & Threshold values \\
\hline From input layer & -2.3002 & 1.5414 & -0.62597 & -0.57284 & -2.3972 \\
to the first hidden & -0.49192 & 2.338 & 2.3407 & -2.4808 & 2.9269 \\
layer & 0.22918 & -2.721 & 0.95017 & 1.2658 & -0.80139 \\
& 3.0541 & -2.6617 & -1.1212 & -1.8284 & 3.1951 \\
\hline $\begin{array}{l}\text { From the first hidden } \\
\text { layer to the second }\end{array}$ & -2.027 & -2.9971 & 1.1971 & 1.0783 & 0.46792 \\
one & 2.3091 & 1.2167 & 2.1645 & 1.9621 & 1.3673 \\
\hline From hidden layer & 2.2576 & 2.1872 & -2.123 & 2.3071 & 1.1887 \\
to output layer & -0.67477 & 0.34548 & -0.057811 & 0.46792 \\
\hline Running time $/ \mathrm{s}$ & 6.5 & & & & \\
\hline Training steps & 10 & & & & \\
\hline
\end{tabular}

Table 3. The prediction results

\begin{tabular}{lllllll}
\hline \multirow{2}{*}{ No. } & \multirow{2}{*}{ Site for Sampling } & Prediction Output & Due Output & \multicolumn{2}{c}{ Outburst risk } \\
\cline { 6 - 6 } & & & Prediction & Test \\
\hline 1 & Luling 82 & 0.025694 & 0 & $\mathrm{I}$ & 1 \\
3 & Kongzhuang $8 \#$ & 0.022138 & 0 & $\mathrm{I}$ & 1 \\
12 & Luling 83 & 1 & 1 & II & II
\end{tabular}




\begin{tabular}{llllll}
18 & Majiagou 9\# & 0.99974 & 1 & II & II \\
24 & Xinzhuangzi & 0.99972 & 1 & II & II \\
27 & Panji No.2 Mine & 0.9997 & 1 & II & II \\
33 & Luling 82 & 1.0001 & 1 & II & II \\
38 & Majiagou 9\# & 0.99984 & 1 & II & II \\
\hline
\end{tabular}

\section{CONCLUSIONS}

Through analyzing the ability of BP algorithm and compound GA-BP algorithm for the ANN model of outburst risk prediction, it is indicated that the running efficiency and predicting effect of compound GA-BP algorithm are obviously improved compared with BP algorithm. By adopting GA-BP compound algorithm, the performance of the network is improved, then the predicting accuracy and reliability of the model is also increased.

\section{ACKNOLEDGEMENT}

This work is supported by National Natural Science Foundation of China (No.50404016, 50534090), Fok Ying Tung Education Foundation (No.101050) and Youth Research Programme of CUMT (No. 0D4789)

\section{REFERENCES}

1. Wang Kai, Yu Qixiang. Non-linear Characteristics and Prediction Model of Coal and Gas Outburst. Xuzhou: China University of Mining and Technology Press, 2005.3 (In Chinese)

2. Wang Kai, Yu Qixiang, Jiang Chenglin, Wu Yagin. Experimental Study on the Intensity of Coal and Gas Outburst. New Development on Rock Mechanics and Engineering: Proceedings of 2nd International Conference on Rock Mechanics and Engineering. Rinton Press, USA, 2002, 227-230

3. The research center of Feisi technology product. MATLAB 6.5 Assistant Analysis and Design of Neural Network. Beijing: Electronics Industry Press, 2003.1 (In Chinese)

4. Yang Xingiun, Zheng Junli. The Artificial Neural Network and Blind Signal Handling. Beijing: TsingHua University Press, 2003.1 (In Chinese)

5. Yuan Ximin, Li Hongyan. Application of Neural Network and Genetic Algorithm in Hydraulic Science. Beijing: China Hydraulic Power Press, 2002.8 (In Chinese) 